\title{
Not Another Missed Spinal Epidural Abscess
}

Loren Gorosh, MD; Stephen Bretz, MD

\section{A 55-year-old man presented for evaluation of a 2-day history of worsening left lower back pain.}

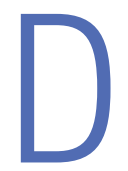
elay in the diagnosis and treatment of spinal epidural abscess (SEA) increases the likelihood of permanent disability (eg, residual motor weakness) or even death. ${ }^{1-5}$ Studies suggest that the incidence of SEA may be on the rise, ${ }^{6,7}$ which is especially troubling in an era of emerging antibiotic resistance. ${ }^{8}$ The pervasive theme among the medical literature stresses the challenges with early recognition; however, missed SEA is a theoretical mishap in a manner akin to Schrödinger's cat or Heisenberg's uncertainty principle. ${ }^{9,10}$

One will recall Erwin Schrödinger's thought experiment of 1935 when he challenged the theory of quantum mechanics by asking whether or not the cat in a box is still alive if there is a 50/50 chance poisonous gas has been released. He suggested that before one looks in the box, the cat is both alive and dead-a state of superposition.

Unfortunately, medical diagnoses do not exist in dual states. Tests are either positive or negative; disease is either present or absent; and in medicine, the cat is either alive or dead. Moreover, when SEA is diagnosed after the initial presentation and workup, (ie, the "bounce-back"), the clinician cannot categorically assume the condition was present, but missed, at the initial evaluation. We present the following case, not as a miraculous catch, or a "zebra-hunting guide" but rather as a rare glimpse into the evolution of a disease process.

\section{Case}

A 55-year-old man with history of type 2 diabetes mellitus (DM), hypertension, and hyperlipidemia presented to the ED with a 2-day history of progressively worsening left lower back pain. Although the patient denied a recent history of trauma, he did state that he helped one of his friends move furniture 1 day prior to presentation and had attributed the worsening pain to this event. The patient described his pain as mild and dull when he was at rest, rating it as a 2 on a pain scale of 1 to 10; and sharp-feeling and at its worst upon movement, rating it as a 9 on a pain scale of 1 to 10 . The patient noted experiencing only mild relief when he shifted to certain positions.

The patient's pain was nonradiating and associated with dull pain in the left ante-

Dr Gorosh is an emergency physician, department of emergency medicine, Swedish Medical Center, Issaquah, Washington; Mill Creek, Washington; and Redmond, Washington. Dr Bretz is an emergency physician, department of emergency medicine, Swedish Medical Center, Issaquah, Washington; Mill Creek, Washington; and Redmond, Washington.

Authors' Disclosure Statement: The authors report no actual or potential conflict of interest in relation to this article.

DOI: 10.12788/emed.2018.0098 
rior proximal thigh. The patient denied any numbness or weakness in any of his extremities. He also denied any perineal numbness or urinary or bowel incontinence; however, he did note experiencing a sense of incomplete evacuation of stools over the past 5 mornings.

The patient denied any recent history of fever, chills, numbness, weakness, difficulty with balance, direct trauma, instrumentation or chiropractic manipulation, or unexplained or unintentional weight loss. He had no history of malignancy and vehemently denied intravenous (IV) drug use.

On physical examination, the patient's vital signs were: blood pressure, 143/93 mm Hg; heart rate, 108 beats/min; respiratory rate, 16 breaths/min; and temperature, $97.6^{\circ} \mathrm{F}$. Oxygen saturation was $95 \%$ on room air. Upon examination, the patient was in no acute distress and was resting comfortably and quietly. Pertinent findings included a supple neck examination, without lymphadenopathy or meningismus. There was no midline tenderness to palpation of the cervical spine and no step-off deformities. The lungs were clear to auscultation bilaterally and without wheezing, rhonchi, or rales. Examination of the heart revealed a regular rhythm with borderline tachycardia, but without murmurs, rubs, or gallops. The patient had 2+ pulses in all four extremities, and capillary refill was less than 2 seconds. The abdomen was soft and nontender, without rebound, guarding, or rigidity. There were no pulsatile abdominal masses or bruits, and bowel sounds were present. The patient had no costovertebral angle tenderness on percussion, and had full range of motion of all four extremities, with no tenderness to palpation and no bony deformities.

Examination of the back revealed a positive straight leg raise on the right, but there was no midline tenderness to palpation or step-off deformity of the thoracic or lumbar spine. The patient exhibited mild leftsided upper lumbar paraspinal tenderness to palpation, but had no associated muscle spasm or overlying skin changes. On neurological assessment, the patient was alert and oriented with cranial nerves II-XII intact. He had 5/5 motor strength in all four extremities, with careful attention to hip flexion and extension, knee flexion and extension, and dorsiflexion and plantar flexion at the ankle. The sensory examination was normal, as were patella and ankle reflexes. The patient was able to ambulate with a steady gait.

Laboratory evaluation included a complete blood count, basic metabolic profile (BMP), urinalysis, erythrocyte sedimentation rate (ESR) and C-reactive protein (CRP) level. The urinalysis was negative for blood or signs of infection. The BMP demonstrated hyperglycemia without acidosis, but no additional electrolyte abnormalities or renal insufficiency. The patient did have leukocytosis (white blood cell [WBC], $19.8 \times 10^{9} / \mathrm{L}$ ) with a left shift. The ESR was within normal limits $(14 \mathrm{~mm} / \mathrm{h})$, but the CRP was mildly elevated (37.33 $\mathrm{nmol} / \mathrm{L}$ ).

Blood cultures were ordered, and the patient was given 2,500 mg vancomycin and 2,000 mg ceftriaxone IV. Given the patient's abnormal back examination and the presence of a leukocytosis, a magnetic resonance imaging (MRI) study of the thoracic and lumbar spine was ordered. Radiology services reported the following findings from the MRI:

n Unremarkable thoracic spine MRI. No evidence of thoracic spine infection or significant degenerative changes.

- No evidence of infection involving the lumbar spine.

- L3-4 and L4-5 disc bulges and posterior element degenerative changes with moderate canal stenoses.

- Edema in the posterior paraspinous musculature on the left at the L3 and L4 levels.

The patient had DM with multiple systemic inflammatory response syndrome criteria and was admitted to the hospital for undifferentiated sepsis, clinical uncer- 
tainty, and pain control. Within 24 hours, blood cultures were positive for gram-positive cocci, later identified as methicillinsensitive Staphylococcus aureus. However, despite treatment with antibiotics and analgesics, the patient's back pain persisted. A repeat MRI of the lumbar spine obtained on hospital day 4 revealed the following:

At L3-4, since the comparison study, there has been development of two epidural abscesses with abnormal peripheral enhancement, one located dorsally measuring 6.8 x 8.1 $\mathrm{x} 14 \mathrm{~mm}$ and another located in the left lateral recess measuring $8.1 \mathrm{x}$ $9.7 \times 10.9 \mathrm{~mm}$. The combination of the broad-based disk bulge, epidural abscesses, and hypertrophic facets resulted in severe spinal canal stenosis.

After receiving this report, the hospitalist contacted neurosurgery services. Shortly thereafter the patient underwent unilateral laminotomy with bilateral canal decompression on hospital day 5 . He was discharged home on hospital day 10 without any neurological deficits, and continued IV antibiotics as an outpatient for an additional 5 weeks.

\section{Discussion}

Only a minority of patients with SEA present with the classic triad of back pain, fever, and progressive neurological findings associated with this condition. ${ }^{1}$ Careful history-taking therefore is essential to identify high-risk patients. Risk factors for SEA include diabetes, IV drug abuse, immunosuppression, chronic renal failure, liver disease, alcoholism, indwelling catheter, recent invasive spinal procedure, recent vertebral fracture, cancer, and distant site of infection. ${ }^{1-3,5,7,11}$

Leukocytosis (WBC $>10 \times 10^{9} / \mathrm{L}$ ) is only found in two-thirds or less of patients with SEA at the time of admission..$^{1,3,12}$
Inflammatory markers such as CRP and ESR are more sensitive but not specific to SEAs. ${ }^{1,2,5,7,11-13}$

An MRI study with gadolinium is the diagnostic modality of choice over computed tomography myelography to assess for SEAs due to its noninvasive nature and ability to better delineate the extent of disease. ${ }^{5,7,14}$ An MRI of the entire spine is recommended to delineate longitudinal and paraspinal extension as SEA can traverse multiple vertebral levels. ${ }^{15}$ While awaiting the results of blood cultures, patients should be treated with broad spectrum antibiotics that include coverage of the most common etiology of SEA, $S$ aureus. , $^{1,3,4,7,11,13}$ While some cases of SEA may be managed medically, the emergency physician should always treat SEA as a neurosurgical emergency and obtain consultation with the appropriate services (eg, neurosurgery, infectious disease, neurology radiology). ${ }^{1,5}$

Our patient represents an unusual case of SEAs in that he presented with $S$ aureus bacteremia while afebrile, along with back pain and tachycardia. He subsequently developed SEA, which was recognized only through serial MRI studies. The patient's tachycardia alone could have been easily attributed to pain and anxiety associated with the ED environment. As such, he could have easily been discharged home with a prescription [for] nonsteroidal antiinflammatory drugs and/or muscle relaxers for pain management-though it is likely that he would have returned to the ED 3 days later with persistent and even worsening symptoms, during which he would have undergone additional testing, possibly MRI, which would have revealed the missed SEA.

Our case clearly demonstrates that no SEA was present at the time of the patient's visit. Thus, the proverbial "missed SEA" may not have been overlooked but rather had not yet developed.

Studies show that half of all patients with SEAs are not diagnosed until after two or more visits to the ED. ${ }^{1,11}$ The literature 
posits that most cases are misdiagnosed at the time of initial evaluation. It has even been postulated that "misdiagnosis of spinal epidural abscess is the rule rather than the exception." Although our patient was eventually diagnosed with a SEA, it was not present on the first MRI taken during the initial evaluation.

\section{Summary}

Unlike the rules of quantum mechanics and the paradox of Schrödinger's cat, SEA follows a progression of disease., ${ }^{3,76}$ There is no superposition-the MRI is either positive or negative. However, excellent care requires the practitioner to know the risk factors of SEA, apply the appropriate screening tests, obtain MRI when necessary, and if diagnostic uncertainty remains, discuss with the patient or family signs and symptoms to monitor as well as reasons to return for re-evaluation.

\section{References}

1. Davis DP, Wold RM, Patel RJ, et al. The clinical presentation and impact of diagnostic delays on emergency department patients with spinal epidural abscess. J Emerg Med. 2004;26(3):285-291.

2. Bhise V, Meyer A, Singh H, et al. errors in diagnosis of spinal epidural abscesses in the era of electronic health records. Am J Med. 2017;130(8):975-981. doi:10.1016/j.amjmed.2017.03.009.

3. Darouiche RO, Hamill RJ, Greenberg SB, Weathers SW, Musher DM. Bacterial spinal epidural abscess. Review of 43 cases and literature survey. Medicine (Baltimore). 1992;71(6):369-385.
4. Baker AS, Ojemann RG, Swartz MN, Richardson EP Jr. Spinal epidural abscess. N Engl J Med. 1975;293(10):463-468. doi:10.1056/ NEJM197509042931001.

5. Nussbaum ES, Rigamonti D, Standiford H, Numaguchi Y, Wolf AL, Robinson WL. Spinal epidural abscess: a report of 40 cases and review. Surg Neurol. 1992;38(3):225-231.

6. Vakili M, Crum-Cianflone NF. Spinal epidural abscess: a series of 101 cases. Am J Med. 2017;130(12):1458-1463. doi:10.1016/j.amjmed.2017.07.017.

7. Rigamonti D, Liem L, Sampath P, et al. Spinal epidural abscess: contemporary trends in etiology, evaluation, and management. Surg Neurol. 1999;52(2):189-196; discussion 197.

8. The World Health Organization. Antimicrobial resistance: global report on surveillance. http://apps.who. int/iris/bitstream/10665/112642/1/9789241564748_ eng.pdf?ua=1. Accessed June 12, 2018.

9. Schrödinger E. Die gegenwärtige situation in der quantenmechanik. Maturwissenschaften. 1935;23(48):807-812; 823-828; 844-849. doi:10.1007/ BF01491891.

10. Heisenberg H. Über quantentheoretische umdeutung kinematischer und mechanischer beziehungen. Zeitschrift für Physik. 1925;33(1):879-893. doi:10.1007/BF01328377.

11. Tang HJ, Lin HJ, Liu YC, Li CM. Spinal epidural abscess-experience with 46 patients and evaluation of prognostic factors. J Infect. 2002;45(2):76-81.

12. Soehle M, Wallenfang T. Spinal epidural abscesses: clinical manifestations, prognostic factors, and outcomes. Neurosurgery. 2002;51(1):79-85; discussion 86-87.

13. Del Curling O Jr, Gower DJ, McWhorter JM. Changing concepts in spinal epidural abscess: a report of 29 cases. Neurosurgery. 1990;27(2):185-192.

14. Hlavin ML, Kaminski HJ, Ross JS, Ganz E. Spinal epidural abscess: a ten-year perspective. Neurosurgery. 1990;27(2):177-184.

15. Parkinson JF, Sekhon LH. Spinal epidural abscess: appearance on magnetic resonance imaging as a guide to surgical management. Report of five cases. Neurosurg Focus. 2004;17(6):E12.

16. Heusner AP. Nontuberculous spinal epidural infections. N Engl J Med. 1948;239(23):845-854. doi:10.1056/NEJM194812022392301. 